\title{
Atomic vibrations in iron nanoclusters: Nuclear resonant inelastic x-ray scattering and molecular dynamics simulations
}

\author{
B. Roldan Cuenya, ${ }^{1}{ }^{*}$ A. Naitabdi, ${ }^{1}$ J. Croy, ${ }^{1}$ W. Sturhahn, ${ }^{2}$ J. Y. Zhao, ${ }^{2}$ E. E. Alp,${ }^{2}$ R. Meyer, ${ }^{3}$ D. Sudfeld,${ }^{3}$ \\ E. Schuster, ${ }^{3}$ and W. Keune $\mathrm{e}^{1,3}$ \\ ${ }^{1}$ Department of Physics, University of Central Florida, Orlando, Florida 32816, USA \\ ${ }^{2}$ Advanced Photon Source, Argonne National Laboratory, Argonne, Illinois 60439, USA \\ ${ }^{3}$ Fachbereich Physik, Universität Duisburg-Essen, D-47048 Duisburg, Germany
}

(Received 1 September 2007; published 15 November 2007)

\begin{abstract}
The lattice vibrational dynamics of supported, self-assembled, isolated ${ }^{57} \mathrm{Fe}$ nanoclusters was studied by nuclear resonant inelastic x-ray scattering and molecular dynamics calculations. The morphological and structural properties and the chemical state of the experimental nanoclusters were investigated by atomic force microscopy, high resolution transmission electron microscopy, and x-ray photoelectron spectroscopy. The measured and calculated vibrational densities of states (VDOSs) reveal an enhancement of the low- and high-energy phonon modes and provide experimental and theoretical proof of non-Debye-like behavior in the low-energy region of the VDOS. Experimentally, this effect was found to depend on the nature of the surface shell (oxide or carbide) of the core/shell nanoclusters. According to the calculations for supported isolated pure Fe nanoclusters, the non-Debye-like behavior appears not only in the surface shell but also in the bcc-Fe core of the nanocluster due to the hybridization of surface and bulk modes.
\end{abstract}

DOI: 10.1103/PhysRevB.76.195422 PACS number(s): 63.22. $+\mathrm{m}$, 02.70.Ns, 61.46. $-\mathrm{w}$, 81.07.Bc

\section{INTRODUCTION}

A profound knowledge of the atomic vibrational properties of nanostructured materials is of great fundamental and technological importance since the vibrational properties, in particular, the vibrational density of states (VDOS), are the key to an understanding of thermodynamic properties such as heat capacity, vibrational entropy, mean sound velocity, Debye temperature, and thermal conductivity, as well as electron-phonon coupling and $1 / f$ noise of electronic devices. ${ }^{1-4}$ Moreover, phonon-assisted chemical reactions might play an important role in some catalytic properties of nanoclusters. ${ }^{5}$ While the vibrational properties of so-called nanocrystalline materials-i.e. "compacted" polycrystalline aggregates with grain sizes in the nanometer regime and dominated by grain boundaries-have been studied extensively, both experimentally ${ }^{6-14}$ and theoretically, ${ }^{15-18}$ there are so far no experimental results available for isolated metallic nanoclusters (NCs), and only few theoretical studies on the VDOS of isolated, unsupported (free) NCs have been reported. ${ }^{19,20}$ Neutron scattering and nuclear resonant inelastic x-ray scattering (NRIXS) experiments ${ }^{6-14}$ as well as computer simulations ${ }^{15-17}$ have revealed clear differences between the VDOS, $g(E)$, of nanocrystalline and bulk metals. In particular, enhancements of $g(E)$ at low and high energies $E$ and a broadening of the VDOS peaks have been observed. The high- $E$ contribution in the experiments is mainly ascribed to phonon lifetime broadening ${ }^{10,12-14}$ and to the presence of surface oxides. ${ }^{12-14}$ Computer simulations ${ }^{15-17}$ have shown that the low- $E$ enhancement of $g(E)$ is mainly due to atoms located in grain boundaries, although oxidation might also contribute to this effect. ${ }^{12-14}$ Nevertheless, the physical nature of the low- $E$ excess modes in nanocrystalline materials is still highly controversial, as linear, ${ }^{11}$ nonlinear, ${ }^{15-17}$ and Debye-like quadratic ${ }^{6-10,12-14}$ behaviors of the low- $E$ VDOS have been reported. In the case of free isolated NCs, theoret- ical calculations have also found excess modes at low phonon energies. ${ }^{19,20}$ However, neither oxidation effects nor grain boundaries can account for the increase of the free NC's VDOS since the calculations were performed for pure defect-free particles. While it is generally assumed that the excess modes are due to surface effects, ${ }^{19,20}$ including lowcoordinated surface atoms, ${ }^{21,22}$ the exact mechanism leading to the VDOS enhancement in free NCs is still a matter of debate. In this paper, we present experimental proof for the existence of low- $E$ excess vibrational modes (and their origin) in supported, isolated, size-selected (single grain) ${ }^{57} \mathrm{Fe}$ core/shell NCs obtained from NRIXS measurements. Supported NCs had to be chosen since free NCs would require measurements of NCs in the gas phase which cannot be realized. In order to compare our experimental results with theory, we have performed molecular dynamics (MD) simulations of supported Fe NCs. These simulations show that the increased low- $E$ VDOS of supported NCs is not a pure surface effect but can also be observed in the local VDOS of the NCs' core.

\section{EXPERIMENTAL DETAILS}

Monolayer-thick films of size-selected, isolated ${ }^{57} \mathrm{Fe} \mathrm{NCs}$ uniformly spaced over large surface areas were prepared using micelle encapsulation ${ }^{23}$ on $\operatorname{PS}(x)-\mathrm{P} 2 \mathrm{VP}(y)$ diblock copolymers (molecular weights: $x=81000$ and $y=14200$ for sample 1 and $x=27700$ and $y=4300$ for sample 2) mixed with ${ }^{57} \mathrm{FeCl}_{3}$ salt. The distinct molecular weights of the two polymers result in samples with different average NC diameters $d$ and intercluster distances $l .{ }^{24}$

The morphological and structural properties of the ${ }^{57} \mathrm{Fe}$ $\mathrm{NCs}$, deposited on $\mathrm{SiO}_{2} / \mathrm{Si}(111)$ wafers and C-coated $\mathrm{Ni}$ grids, were investigated by atomic force microscopy (AFM) and high resolution transmission electron microscopy 
(HRTEM). NRIXS measurements on the $\mathrm{SiO}_{2} / \mathrm{Si}(111)$ supported ${ }^{57} \mathrm{Fe}$ NCs (samples 1, 2, and $2 \mathrm{~b}$ ) were performed at room temperature in air at beamline 3-ID at the Advanced Photon Source by scanning the synchrotron-beam energy around the resonant energy of $14.413 \mathrm{keV}$ of the ${ }^{57} \mathrm{Fe}$ nucleus with an energy resolution of $1.3 \mathrm{meV}$. The measurement time per sample was two days. The method is described in Refs. $25-29$. Using the PHOENIX software, ${ }^{28}$ the spectra have been decomposed into single-phonon $[\propto g(E)]$ and multiphonon contributions.

\section{THEORETICAL DETAILS}

MD simulations of bulk bcc-Fe and hemispherical $\mathrm{Fe}$ clusters supported on a $\operatorname{Ag}(001)$ substrate provided their VDOS. The interatomic forces were calculated from embedded-atom method (EAM) potentials.$^{30}$ For the Fe atoms, the potential described in Ref. 31 was used together with a similarly constructed Ag potential. Realistic model configurations of deposited NCs were generated from simulations of energetic cluster impacts: two spherical bcc-Fe NCs containing 749 and 5577 atoms were deposited on a $\mathrm{Ag}(001)$ substrate with impact energies of $1 \mathrm{eV}$ per atom. The simulations were continued until the deposited NCs, which melted during the deposition, had completely recrystallized. Finally, the VDOS of the resulting supported NCs with diameters of 3.1 and $7.3 \mathrm{~nm}$ were calculated from the Fourier transform of their velocity-autocorrelation functions, and local crystalline structures were identified with the help of a common-neighbor analysis (CNA) ${ }^{32}$

\section{RESULTS AND DISCUSSION}

Figure 1 shows AFM images from ${ }^{57} \mathrm{Fe}$ NCs deposited on $\mathrm{SiO}_{2} / \mathrm{Si}(111)$ obtained after polymer removal by annealing in ultrahigh vacuum (UHV) at $773 \mathrm{~K}$ [(a) sample $1 \mathrm{~b}$, (c) sample 2b] and subsequent in situ $\mathrm{Ar}^{+}$etching $(1 \mathrm{keV})$ and air exposure [(b) sample 1, (d) sample 2]. Isolated, monodispersed NCs with local hexagonal arrangement can be observed. On the $\mathrm{Ar}^{+}$-etched samples, a minimum residual $\mathrm{C}$ signal, comparable to the one obtained upon annealing a polymer-free Si substrate (coated by adventitious carbon) under UHV, was observed by x-ray photoelectron spectroscopy (XPS) (not shown). The $\mathrm{Ar}^{+}$etching did not result in distortions of the NC's spatial arrangement. It did, however, provide a partial ultrathin Si coating, presumably along the NC's rim, that minimized the oxidation of the NCs upon air exposure, as evidenced by XPS. The $\mathrm{Ar}^{+}$-etched samples appeared to be more resistant to oxidation, since after 7 months in air, the XPS spectra $(\mathrm{Al} K \alpha=1486.6 \mathrm{eV})$ ) from sample 2 showed $30.5 \% \mathrm{Fe}^{0}$ and $69.5 \% \mathrm{Fe}^{3+}$ [Fig. 2(b), curve (vi)]. On a similarly synthesized sample (sample 1), after sputtering and a long air exposure (7 months), $16 \% \mathrm{Fe}^{0}$ and $84 \% \mathrm{Fe}^{3+}$ were detected [Fig. 2(a), curve (vi)]. These results indicate that sample 2 has the lowest concentration of $\mathrm{Fe}^{3+}$ (or the thinnest oxide shell). Scanning tunneling microscopy (STM) images were also acquired on these samples. We found values of $d=9.8 \pm 0.6$ and $8.2 \pm 0.6 \mathrm{~nm}(\mathrm{STM})$ and $l=70 \pm 11$ and $37 \pm 3 \mathrm{~nm}(\mathrm{AFM})$ for samples 1 and 2 , respectively. The $\mathrm{NCs}$

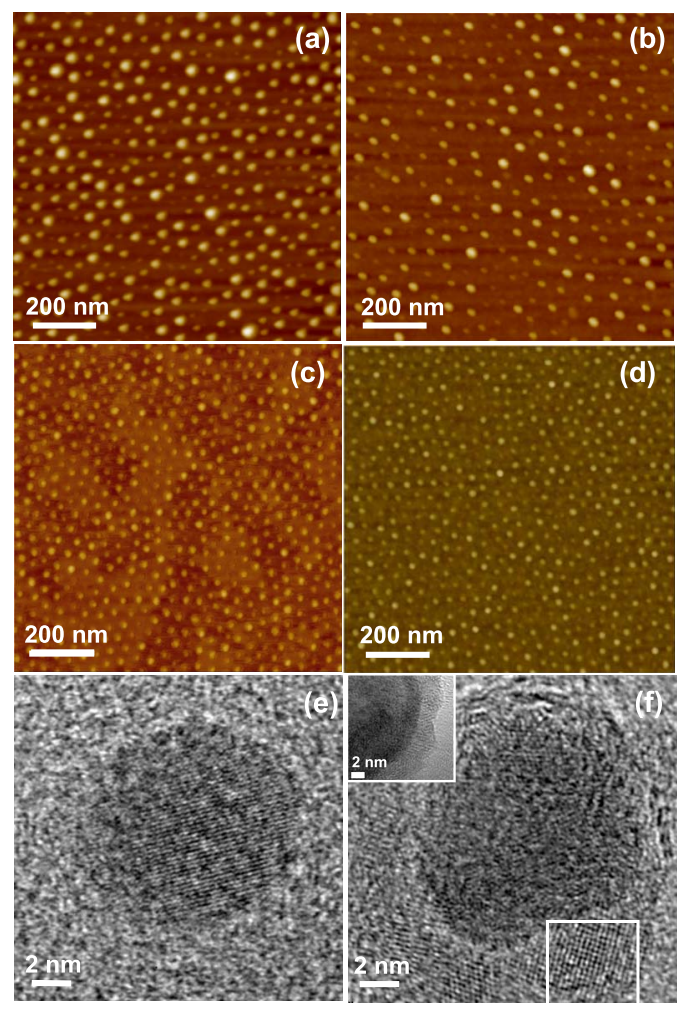

FIG. 1. (Color online) $[(\mathrm{a})-(\mathrm{d})] \mathrm{AFM}$ and $[(\mathrm{e})$ and (f)] high resolution TEM images of ${ }^{57} \mathrm{Fe}$ nanoclusters synthesized by encapsulation in PS(81000)-P2VP(14200) [top row and (e)] and PS(27700)-P2VP(4300) [center row and (f)]. The nanoclusters were deposited on $\mathrm{SiO}_{2} / \mathrm{Si}(111)$ (for AFM) and on C-coated Ni grids (for TEM). The images were obtained after polymer removal by annealing in UHV to $773 \mathrm{~K}$ for $30 \mathrm{~min}$ [(a), (c), and (f) for sample 2b] and after in situ sputtering [(b) and (e) for sample 1 and (d) for sample 2]. The top inset in the TEM image (f) demonstrates the core-shell structure of the nanoclusters in sample $2 \mathrm{~b}$. Both shell and core are crystalline. A FFT analysis from the region marked in (f) with a square frame (bottom right corner) has been conducted.

were found to be nonspherical, with a diameter (STM) to height (AFM) ratio ranging from 2.5 to 5.5. HRTEM measurements reveal that all NCs have a crystalline core [Figs. 1(e) and 1(f)]. The oxide shell could not be detected by HRTEM, probably because it is amorphous and rather thin. From the fast Fourier transform analysis of the TEM image of sample 1, Fig. 1(e), a lattice parameter of $a$ $=0.289 \pm 0.024 \mathrm{~nm}$ corresponding to bcc iron was determined. A third sample (sample $2 \mathrm{~b}$ ) (with $d=8.5 \pm 0.6 \mathrm{~nm}$ and $l=32 \pm 4 \mathrm{~nm}$ ), prepared in a similar way as sample 2 but without $\mathrm{Ar}^{+}$etching after UHV annealing, revealed a crystalline bcc-Fe core/shell structure by HRTEM [Fig. 1(f)], with an $\sim 2-5 \mathrm{~nm}$ thick crystalline shell of (most likely) $\mathrm{Fe}_{3} \mathrm{C}$, according to the lattice spacings. The lattice constant of $a$ $=0.293 \pm 0.024 \mathrm{~nm}$ measured for the core region of the particle in sample $2 \mathrm{~b}$ belongs to the bcc phase of iron [Fig. 1(f)]. The thick shell (between 2 and $5 \mathrm{~nm}$ ) [Fig. 1(f), top inset] has a cubic structure. A fast Fourier transform (FFT) pattern analysis taken from the bright-field image [FFT region of interest is marked in Fig. 1(f), bottom inset] revealed lat- 


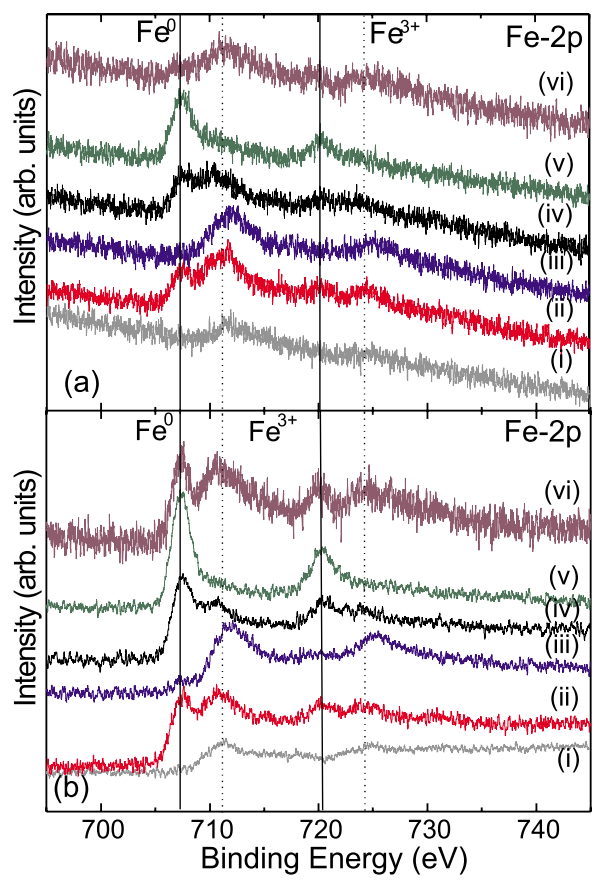

FIG. 2. (Color online) XPS spectra from the Fe $2 p$ core level of size-selected ${ }^{57} \mathrm{Fe}$ nanoclusters deposited on $\mathrm{SiO}_{2} / \mathrm{Si}(111)$. The particles were synthesized by encapsulation on (a) PS(81000)P2VP(14200) and (b) PS(27700)-P2VP(4300) and measured (i) as deposited, (ii) after annealing in UHV at $773 \mathrm{~K}$ for $30 \mathrm{~min}$, and (iii) after subsequent air exposure (one day) [(b) sample 2b], (iv) followed by $\mathrm{Ar}^{+}$sputter etching at $1 \mathrm{keV}$ for 20 min and (v) additional $\mathrm{Ar}^{+}$sputter etching at $1 \mathrm{keV}$ for $60 \mathrm{~min}$. After 7 months of air exposure of the $\mathrm{Ar}^{+}$-etched samples, spectra (vi) were measured [(a) sample 1 and (b) sample 2].

tice spacings of $d_{h k l}=0.252 \pm 0.017 \mathrm{~nm}$ and $d_{h k l}=0.263$ $\pm 0.017 \mathrm{~nm}$. It is most probable that these lattice spacings belong to the (311) orientation of $\mathrm{Fe}_{2} \mathrm{O}_{3}$ or $\mathrm{Fe}_{3} \mathrm{O}_{4}$ or to the (020) orientation of $\mathrm{Fe}_{3} \mathrm{C}$. Since none of the other samples showed this type of shell after similar air exposure, the shell seems to originate from residual polymer that was not removed during the in situ annealing of sample $2 \mathrm{~b}$ and, most likely, is $\mathrm{Fe}_{3} \mathrm{C}$.

Figure 3(a) shows $g(E)$ of the samples 1, 2, and $2 \mathrm{~b}$ and of a reference bulk bcc ${ }^{57} \mathrm{Fe}$ foil obtained from the measured data. The results reveal three clear differences between $g(E)$ of supported isolated Fe NCs and that of a bulk bcc-Fe reference sample: (i) the strong suppression of the sharp phonon peaks, in particular, of the strong longitudinal acoustic phonon peak at $36 \mathrm{meV}$ (this effect may originate from phonon damping due to confinement, ${ }^{10-12}$ ) (ii) the significant enhancement of $g(E)$ at low and high energies, where excitations extend beyond the cut-off energy of $\sim 40 \mathrm{meV}$ of bulk bcc Fe, and (iii) the striking non-Debye-like behavior of $g(E)$ [with $g(E) / E^{2} \neq$ const] of NCs at low energies [see also Fig. 4(a)]. Samples 1 and 2 show a distinct shoulder in the VDOS at low $E$ with a plateau between 6.5 and $14 \mathrm{meV}$ and a common peak at $\sim 41-43 \mathrm{meV}$. It is worthwhile mentioning that bulk hematite $\left(\alpha_{-}{ }^{57} \mathrm{Fe}_{2} \mathrm{O}_{3}\right)$ also shows a peak near $\sim 41 \mathrm{meV} .{ }^{29}$ However, since the strong VDOS peaks of bulk
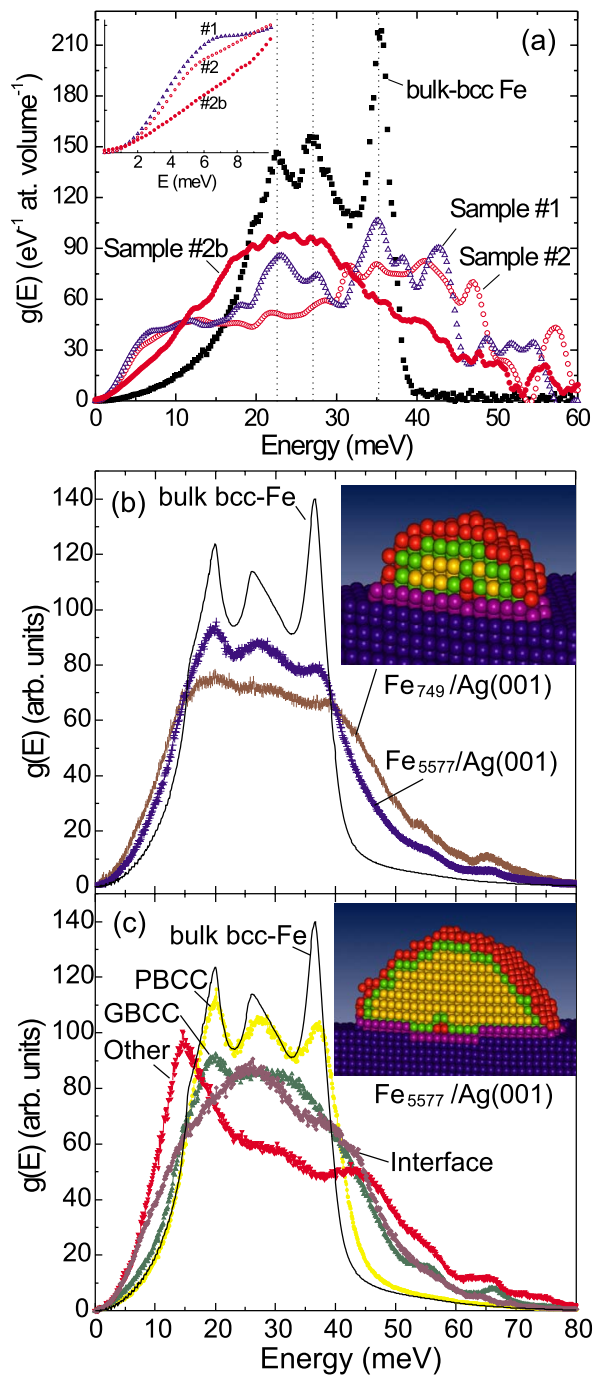

FIG. 3. (Color online) (a) VDOS $[$ or $g(E)]$ of ${ }^{57} \mathrm{Fe}$ nanoclusters in sample 1 (open triangles), sample 2 (open circles), and sample $2 \mathrm{~b}$ (full circles) and of bulk bcc ${ }^{57} \mathrm{Fe}$ (reference, full squares) obtained from NRIXS. Vertical dashed lines: Phonon peak positions of bulk bcc Fe. Inset: Low-energy part of $g(E)$. (b) Total VDOS calculated for bulk bcc-Fe (full black line) and epitaxial bcc-Fe ${ }_{749}$ (brown) and $\mathrm{Fe}_{5577}$ (blue) clusters on $\mathrm{Ag}(001)$. (c) Local VDOS calculated for different $\mathrm{Fe}$ atoms within an $\mathrm{Fe}_{5577}$ cluster on $\mathrm{Ag}(001)$ (see text). All curves are normalized to the same area. Insets: Calculated geometrical arrangement of bcc- $\mathrm{Fe}(001)$ clusters of 749 atoms in (b) and of 5577 atoms in (c) on $\operatorname{Ag}(001)$.

hematite around $\sim 20 \mathrm{meV}$ are missing in the VDOS of our NCs [Fig. 3(a)], it is unclear whether or not the oxide shell of our NCs may be associated with $\alpha-\mathrm{Fe}_{2} \mathrm{O}_{3}$. Oxidized nanocrystalline $\mathrm{Fe}$ does not exhibit such a distinct peak near 41-43 meV. ${ }^{14}$ Although the nature of the $41-43 \mathrm{meV}$ peak is unknown, it seems to be related to some vibrational Fe state in our NC oxide shell that is similar to the $41 \mathrm{meV}$ vibrational mode in bulk $\alpha-\mathrm{Fe}_{2} \mathrm{O}_{3}$. In contrast to samples 1 and 2 , sample $2 \mathrm{~b}$ shows no distinct high-energy peaks and the low-energy region is nearly linear in $E$.

Figure 3(b) shows the $g(E)$ resulting from the MD simulations. The experimental $g(E)$ of bulk bcc Fe [Fig. 3(a)] is 


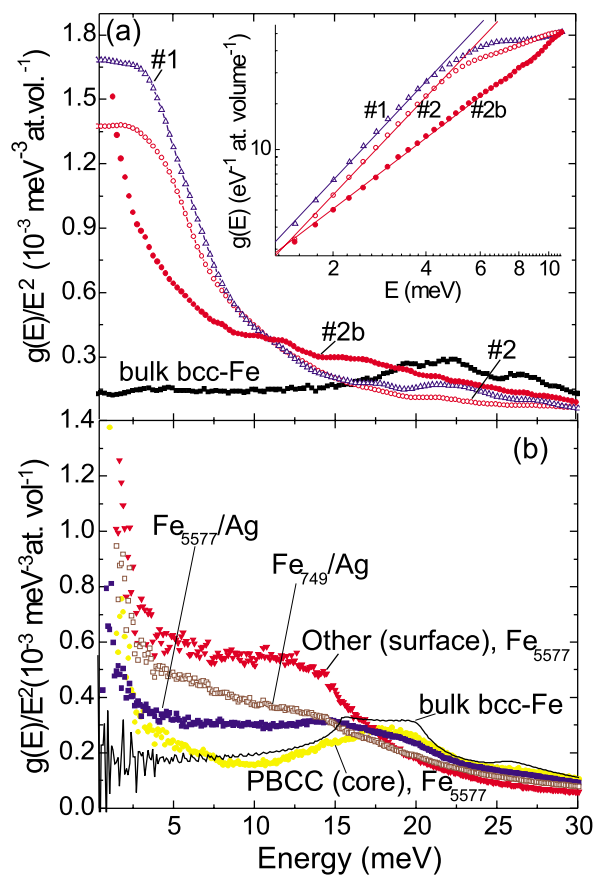

FIG. 4. (Color online) (a) Measured reduced VDOS, $g(E) / E^{2}$, for samples 1, 2, and $2 \mathrm{~b}$ and bulk bcc Fe. (b) Calculated $g(E) / E^{2}$ of bulk bcc-Fe (solid line), $\mathrm{Fe}_{749}$ (open squares), and $\mathrm{Fe}_{5577}$ (full squares) clusters on $\mathrm{Ag}(001)$ and spatially resolved $g(E) / E^{2}$ of $\mathrm{PBCC}$ (core) (full circles) and Other (surface) (full triangles) atoms in an $\mathrm{Fe}_{5577}$ cluster.

fairly well reproduced, although the phonon peak at $36 \mathrm{meV}$ is slightly broadened, probably due to anharmonic effects of the EAM potentials. In agreement with our experimental observations, an increased $g(E)$ was obtained for the NCs at low and high $E$. However, particularly in the low- $E$ regime, the increase is less pronounced than in the experiments. We attribute this difference to the oxide shell (samples 1 and 2) or $\mathrm{Fe}_{3} \mathrm{C}$ shell (sample 2b) of the experimental NCs and, in particular, to differences in the low-coordinated interfacial core/shell or shell surface states. ${ }^{21,22}$ The apparent suppression of the sharp phonon peaks demonstrates a sizedependent phonon confinement in the supported NCs.

Based on a CNA, different types of Fe atoms have been identified and are depicted by different colors in the insets of Figs. 3(b) and 3(c): perfect bcc (PBCC, atoms with bcc environment up to the seventh neighbor shell, yellow), good bcc (GBCC, atoms with bcc environment up to the second neighbor shell, green), interface atoms (Interface, Fe atoms with at least one $\mathrm{Ag}$ atom in the first two neighbor shells, magenta), and "other" Fe atoms (red). In Figs. 3(b) and 3(c), it can be seen that the core of the NCs consists of PBCC atoms, whereas the GBCC atoms form a subsurface layer between the surface (made of "Other" atoms) and the core. Figure 3(c) shows the VDOS contributions of the different atom types normalized to the total VDOS of Fe atoms in the simulated $\mathrm{Fe}_{5577} \mathrm{NC}$. Figure 3(c) shows that the largest contribution to the VDOS enhancements, at high and low $E$, originates from surface (Other), Interface, and GBCC atoms, whereas the PBCC core of the NCs behaves nearly bulklike, except for $E<4-5 \mathrm{meV}$ (see below).
A striking feature measured for our supported, isolated ${ }^{57} \mathrm{Fe} \mathrm{NCs}$ is the anomalous nonquadratic behavior in the VDOS at low $E$ [Fig. 4(a)]. The log-log plot of $g(E) \propto E^{n}$ provides $n=1.86 \pm 0.03(E \leqslant 4.8 \mathrm{meV}$, sample 1$), n=1.84$ $\pm 0.02(E \leqslant 5.0 \mathrm{meV}$, sample 2), and $n=1.33 \pm 0.01 \quad(E$ $\leqslant 10 \mathrm{meV}$, sample 2b) [Fig. 4(a), inset]. These values are all below $n=2$, and sample $2 \mathrm{~b}$ is closer to two-dimensional (2D) behavior $(n=1)$ than to Debye behavior $(n=2)$. The VDOS derived from our MD simulations also shows non-Debye behavior, not only for Fe atoms at the surface but also in the core [Fig. 3(b)]. However, the effect [Fig. 4(b)] is much less pronounced than in the experimental systems [Fig. 4(a)]. Again, this difference can be attributed to the oxide shell (samples 1 and 2) or $\mathrm{Fe}_{3} \mathrm{C}$ shell (sample 2b). We therefore conclude, in agreement with Ref. 20, that the formation of an outer cluster shell leads to a significant enhancement of the anomalous VDOS behavior at low energies. This is further confirmed by the fact that, for a given shell, the number of observed low- $E$ phonon modes scales with the NC's shell thickness [Fig. 3(a), inset]. The samples with the thickest (sample 1) and thinnest (sample 2) oxide shells (according to our XPS data) show the highest and lowest $g(E)$ enhancements, respectively. Obviously, the nature of the shell (oxide or carbide) affects the type of non-Debye behavior at low $E$, presumably via differences in low-coordinated interfacial or surface Fe atoms. ${ }^{21,22}$ Remarkably, the best qualitative agreement is found between sample $2 b$ (carbide shell) and the calculated $\mathrm{Fe}_{749} / \mathrm{Ag}$ (Fig. 4). The carbide shell seems to leave the Fe-core vibrations roughly undisturbed, with the exception of a shift of the non-Debye region at low $E$ from $E<\sim 4 \mathrm{meV}\left(\mathrm{Fe}_{749} / \mathrm{Ag}\right.$ ) to $E<\sim 10 \mathrm{meV}$ (sample $2 \mathrm{~b}$ ).

It is interesting to observe in Fig. 4(b) that $g(E) / E^{2}$ of the PBCC core of the NCs follows closely the behavior of bulk bcc $\mathrm{Fe}$ down to $E \sim 4-5 \mathrm{meV}$, where non-Debye behavior suddenly appears simultaneously in the core and at the surface. We attribute this effect to phonon confinement. The phonon wavelength at this energy can be estimated to be $\lambda$ $\sim 5 \mathrm{~nm}$, which is of the order of our NC size. Therefore, phonon modes with energies below 4-5 meV do not fit into the core alone and both surface and core participate in the vibrations. The parallel increase of $g(E) / E^{2}$ for the core and surface below $\sim 4-5 \mathrm{meV}$ thus indicates hybridization of the NC's core and surface modes.

We would like to emphasize that Debye behavior $(n \approx 2)$ was reported for partially oxidized compacted nanocrystalline Fe. ${ }^{6-10,12-14}$ This is not surprising because such a compacted powder material is inherently a three-dimensional system, contrary to the case of our self-organized arrays of isolated nanoclusters with their large surface-to-volume ratio. The latter property implies low-coordinated interfacial core/shell and/or shell surface states, which, according to theory, ${ }^{19-22}$ lead to the tendency of $2 \mathrm{D}$ behavior in isolated nanoclusters.

\section{CONCLUSIONS}

In summary, our measured and calculated VDOSs of supported, isolated single-grain ${ }^{57} \mathrm{Fe}$ nanoclusters reveal an enhancement of the low- and high- $E$ phonon modes and non- 
Debye behavior in the low- $E$ region. Experimentally, the non-Debye behavior was found to depend on the nature of the NC's surface shell. It reveals a tendency toward $2 \mathrm{D}$ vibrational behavior, very likely due to the high number of low-coordinated interfacial core/shell and/or shell surface states. Furthermore, our calculations show that the nonDebye behavior extends from the surface to the core of the supported NCs due to hybridization of surface and bulk modes. We suppose that our conclusions are not limited to Fe nanoclusters, but are valid for metal NCs in general. The modified VDOS observed for NCs is expected to affect their thermal stability and other temperature-related properties such as chemical order-disorder transitions and the preexponential factor in Arrhenius-type surface phenomena.

\section{ACKNOWLEDGMENTS}

This work was supported by NSF-CAREER (No. 0448491), ACS-PRF (No. 42701-G5), UCF, and DFG (GRK 277, SFB 445, and SFB 491). Use of the APS was supported by DOE (No. W-31-109-EGN-38). *roldan@physics.ucf.edu

${ }^{1}$ A. Kara and T. Rahman, Surf. Sci. Rep. 56, 159 (2005).

${ }^{2}$ Phonons in Nanostructures, edited by M. A. Stroscio and M. Dutta (Cambridge University Press, Cambridge, 2001).

${ }^{3}$ G. P. Srivastava, The Physics of Phonons (Adam Hilger, Bristol, 1990).

${ }^{4}$ M. Mihaila, in From Noise in Communication Systems to Number Theory, Lecture Notes in Physics, edited by M. Planat (Springer, Heidelberg, 2000).

${ }^{5}$ B. Gumhalter and T. Matsushima, Surf. Sci. 561, 183 (2004).

${ }^{6}$ B. Fultz, L. Anthony, L. Nagel, R. Nicklow, and S. Spooner, Phys. Rev. B 52, 3315 (1995).

${ }^{7}$ J. Trampenau, K. Bauszus, W. Petry, and U. Herr, Nanostruct. Mater. 6, 551 (1995)

${ }^{8}$ B. Fultz, J. L. Robertson, T. Stephens, L. Nagel, and S. Spooner, J. Appl. Phys. 79, 8318 (1996).

${ }^{9}$ A. B. Papandrew, A. F. Yue, B. Fultz, I. Halevy, W. Sturhahn, T. S. Toellner, E. E. Alp, and H. Mao, Phys. Rev. B 69, 144301 (2004).

${ }^{10}$ B. Fultz, C. C. Ahn, E. E. Alp, W. Sturhahn, and T. S. Toellner, Phys. Rev. Lett. 79, 937 (1997).

${ }^{11}$ U. Stuhr, H. Wipf, K. H. Andersen, and H. Hahn, Phys. Rev. Lett. 81, 1449 (1998).

${ }^{12}$ H. Frase, B. Fultz, and J. L. Robertson, Phys. Rev. B 57, 898 (1998).

${ }^{13}$ E. Bonetti, L. Pasquini, E. Sampaolesi, A. Deriu, and G. Cicognani, J. Appl. Phys. 88, 4571 (2000).

${ }^{14}$ L. Pasquini, A. Barla, A. I. Chumakov, O. Leupold, R. Rueffer, A. Deriu, and E. Bonetti, Phys. Rev. B 66, 073410 (2002).

${ }^{15}$ R. Meyer, L. J. Lewis, S. Prakash, and P. Entel, Phys. Rev. B 68, 104303 (2003).
${ }^{16}$ P. M. Derlet, R. Meyer, L. J. Lewis, U. Stuhr, and H. V. Swygenhoven, Phys. Rev. Lett. 87, 205501 (2001).

${ }^{17}$ P. M. Derlet and H. Van Swygenhoven, Phys. Rev. Lett. 92, 035505 (2004).

${ }^{18}$ R. Singh and S. Prakash, Surf. Sci. 532-535, 272 (2003).

${ }^{19}$ A. Kara and T. S. Rahman, Phys. Rev. Lett. 81, 1453 (1998).

${ }^{20}$ D. Y. Sun, X. G. Gong, and X. Q. Wang, Phys. Rev. B 63, 193412 (2001).

${ }^{21}$ H. Yildirim, Surf. Sci. 600, 484 (2006).

${ }^{22}$ S. Durukanoglu, A. Kara, and T. S. Rahman, Phys. Rev. B 67, 235405 (2003).

${ }^{23}$ J. P. Spatz, S. Mossmer, C. Hartmann, M. Möller, T. Herzog, M. Krieger, H. G. Boyen, P. Ziemann, and B. Kabius, Langmuir 16, 407 (2000).

${ }^{24}$ For a description of the preparation method, see B. Roldan Cuenya, S. H. Baeck, T. F. Jaramillo, and E. W. McFarland, J. Am. Chem. Soc. 125, 12929 (2003).

${ }^{25}$ M. Seto, Y. Yoda, S. Kikuta, X. W. Zhang, and M. Ando, Phys. Rev. Lett. 74, 3828 (1995).

${ }^{26}$ W. Sturhahn, T. S. Toellner, E. E. Alp, X. Zhang, M. Ando, Y. Yoda, S. Kikuta, M. Seto, C. W. Kimball, and B. Dabrowski, Phys. Rev. Lett. 74, 3832 (1995).

${ }^{27}$ A. I. Chumakov, R. Rüffer, H. F. Grunsteudel, G. Grubel, J. Metge, and H. A. Goodwin, Europhys. Lett. 30, 427 (1995).

${ }^{28}$ W. Sturhahn, Hyperfine Interact. 125, 149 (2000).

${ }^{29}$ A. I. Chumakov and W. Sturhahn, Hyperfine Interact. 123-124, 781 (1999).

${ }^{30}$ M. S. Daw and M. I. Baskes, Phys. Rev. B 29, 6443 (1984).

${ }^{31}$ R. Meyer and P. Entel, Phys. Rev. B 57, 5140 (1998).

${ }^{32}$ D. J. Honeycutt and H. C. Andersen, J. Phys. Chem. 91, 4950 (1987). 